\title{
STUDY AND IMPLEMENTATION OF DC DRIVE USING PIC16F877A MICROCONTROLLER
}

\author{
Pathik A. Kapadiya ${ }^{1}$, Ishan R. Shah ${ }^{2}$, Nirav D. Mehta ${ }^{3}$ \\ ${ }^{I}$ UG Student, Department of Power Electronics, Vishwakarma Government Engineering College, Ahmedabad, Gujarat, \\ India \\ ${ }^{2}$ UG Student, Department of Power Electronics, Vishwakarma Government Engineering College, Ahmedabad, Gujarat, \\ India \\ ${ }^{3}$ Assistant Professor, Department of Power Electronics, Vishwakarma Government Engineering College, Ahmedabad, \\ Gujarat, India
}

\begin{abstract}
In recent world, electrical equipment's like DC motor, AC motor, transformer etc. are the most common devices. In many industrial as well as domestic applications, the dc motors are needed to be operated either on variable speed or constant speed along with its forward and reverse operation. In this paper, the digital controller based control for DC drives is presented for the effective control of the output, when there is a sudden change in the input parameters. An assembly language program has been built for the programmable PIC16F877A microcontroller which controls the various functions of DC drive. The main objective of control is to get the desired output and keep the motor or drive safe in case of any fault occurred. Initially microcontroller has been used for the gating purpose for power electronics switches. Introducing a microcontroller based scheme facilitates the new DC drive system to deal with the various changes in the system and helps in maintaining the safe operation of the system.
\end{abstract}

Keywords-DCMotor,Microcontroller,PWMtechnique,Dutycycle

\section{INTRODUCTION}

Converter-fed separately excited DC motors are widely used in industrial applications requiring a wide range of speed control and/or frequent starting, breaking, and reversing. The continuous operation of a motor is required in some applications like rolling mills, printing press, mine winders etc. For that the motor is needed to be operated with desired speeds for different time periods along with forward and reverse motion. To control such parameters of the motor, the drive circuit is employed either by analog means or digital means. The microcontroller or processor based systems have gained prominence. They have certain inherent advantages over their analog counterparts like higher accuracy, drift free performance, better noise immunity, etc. In addition, they also afford flexibility, provide fault diagnostics, and facilitate easy trouble shooting. Initially the microcontroller can be programed to generate the firing pulses for power electronics switching devices like MOSFET or IGBT. The program can be developed further for controlling various parameters of the DC motor like acceleration and deceleration, forward and reverse operation etc. These all controlling depends on the sampling signals which are measured from the DC motor the armature voltage of the motor and field current of the motor [1]. Apart from this, some of the unwanted conditions like short circuit, under voltage, over current, earthing cause hazardous effects on the motor system. So that the microcontroller based protection scheme for the motor can also be developed to overcome such faulty conditions.

Thus, the robust and multi-purpose peripheral circuit system can be obtained using the intelligent microcontroller IC. Also the supervisory control for all the essential parameter can be obtained with the help of a single microcontroller IC. [2]

\section{CONVENTIONAL DC DRIVE}

In modern electrical drive system, about $50 \%$ of the total energy is consumed by the drives which may be either constant speed drives or variable speed drives. The $75 \%$ of the system has application with constant speed drives in which energy is wasted across the load like throttling valve or any other parameter of interest. Using solid state control of drives, machine speed is adjusted in such a way that it delivers the required flow rate or any other parameter of interest efficiently.

The demerits associated in the system for implementing other performance requirements with such system are: system becomes bulky, inefficient and inflexible to the change parameters. It is clear that the losses are much more in constant speed drives as compared to the variable speed drives. In conventionally analog control scheme, implementation is done by the hardwired electronics. Also the 
non-linearity of speed sensors poses the problem in efficient control of speed of dc drives. The demerits further associated with such control are its temperature dependency and requirement of major changes. [2]

Earlier when power electronics converters were not available, the ac motor and dc generator were coupled to drive the $\mathrm{dc}$ motor. On contrary to this the microcomputer based control has the merits of software implementation which in turn makes the system reliable and flexible in nature. The control is precise and faster in operation as it depends on the programming for the microcontroller.

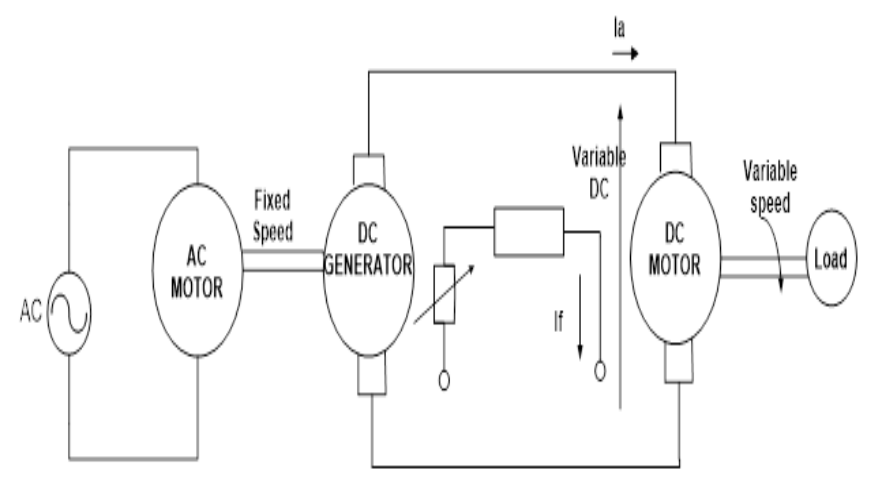

Fig 1 conventional de drive

Also the numbers of analog ICs are reduced due to software implementation by means of some instruction sets. Thus, in recent era, it is more advisable and convenient to use the microcontroller for motor operating system. PIC microcontroller is employed for the motor control operation frequently.

\section{MICROCONTROLLER BASED SINGLE PHASE DC DRIVE}

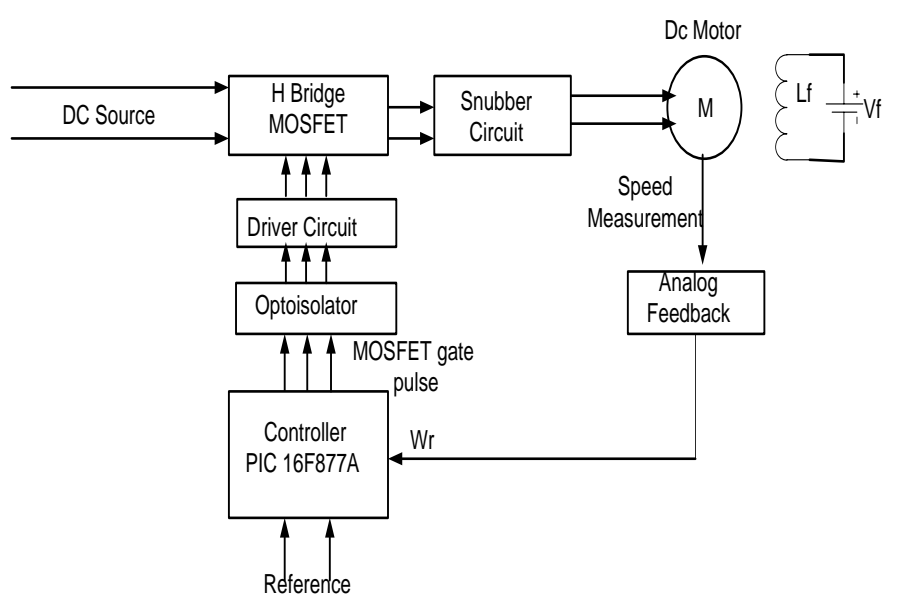

Fig 2 basic block diagram of microcontroller based dc drive
The main functional blocks are as follows:

$>$ Power electronics modulator (H Bridge MOSFET)

$>$ Speed measurement

$>$ Controller circuit

$>$ Driver circuit

$>$ Snubber circuit

The block diagram for single-phase DC motor drive using microcontroller is shown in the

Fig 2. Thus the DC supply is needed for the drive. The power circuit contains microcontroller based pulse generation for triggering of power devices used in converters. Here the MOSFET H-bridge is employed. The control technique is of closed loop type. The microcontroller unit controls the DC motor by processing its controlling parameters that are speed and current signals. In closed feedback loop system, the motor voltage magnitude and polarity can be controlled. Similarly the motor current magnitude and polarity can be controlled. Therefore the motor is allowed to be operated in all four quadrants of V-I plane. [3] Pushbuttons as well as LCD screen is also interfaced here to set various parameters for motor operation like: Acceleration and Deceleration in step speed of the motor.

\section{H BRIDGE CONFIGURATION}

$\mathrm{H}$ Bridge is electronic power circuit that allows a motor speed and direction to be controlled.Often motors are controlled from some kind of "brain" or microcontroller to accomplish a mechanical goal.The microcontroller provides a instructions to the motor but it can not provide the power required to drive the motor.An $\mathrm{H}$ Bridge circuit inputs a microcontroller instruction and amplifies them to drive a mechanical motor.The $\mathrm{H}$ Bridge take a small electrical signal and translate it into high power output for the mechanical motor.[4]

The Basic operating mode of an H-Bridge is fairly simple: If Q3 and Q2 are turned on, the left lead of the motor will be connected to ground, while the right lead is connected to the power supply. Current starts following through the motor which energizes the motor in forward direction and the motor shaft starts spinning. If Q1 and Q4 are turned on, the converse will happen, the motor get energies in the reverse direction, and the shaft will start spinning that way. If less than full speed operation is intended both of the switches are controlled with PWM fashion. The average voltage seen by the motor will be determined by the ration between $\mathrm{ON}$ and $\mathrm{OFF}$ time of the PWM signal.[4] 


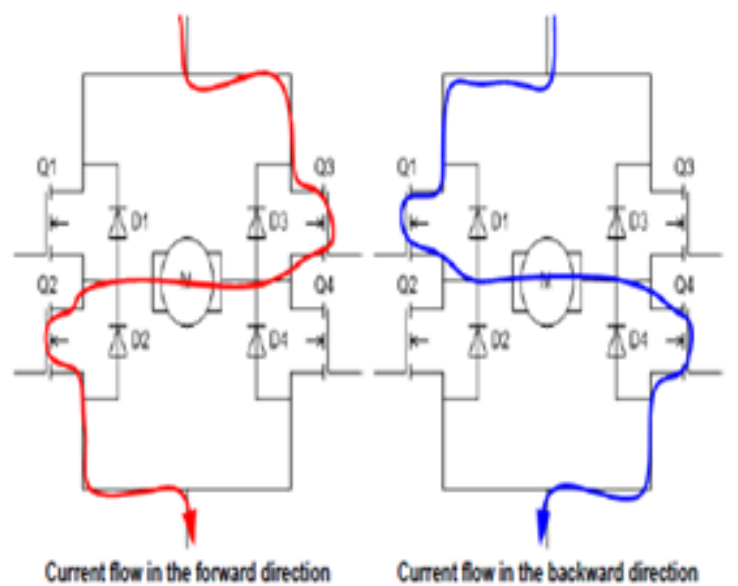

Fig 3 conducting mosfet in forward and reverse direction[4]

\section{SIMULATION OF H BRIDGE}

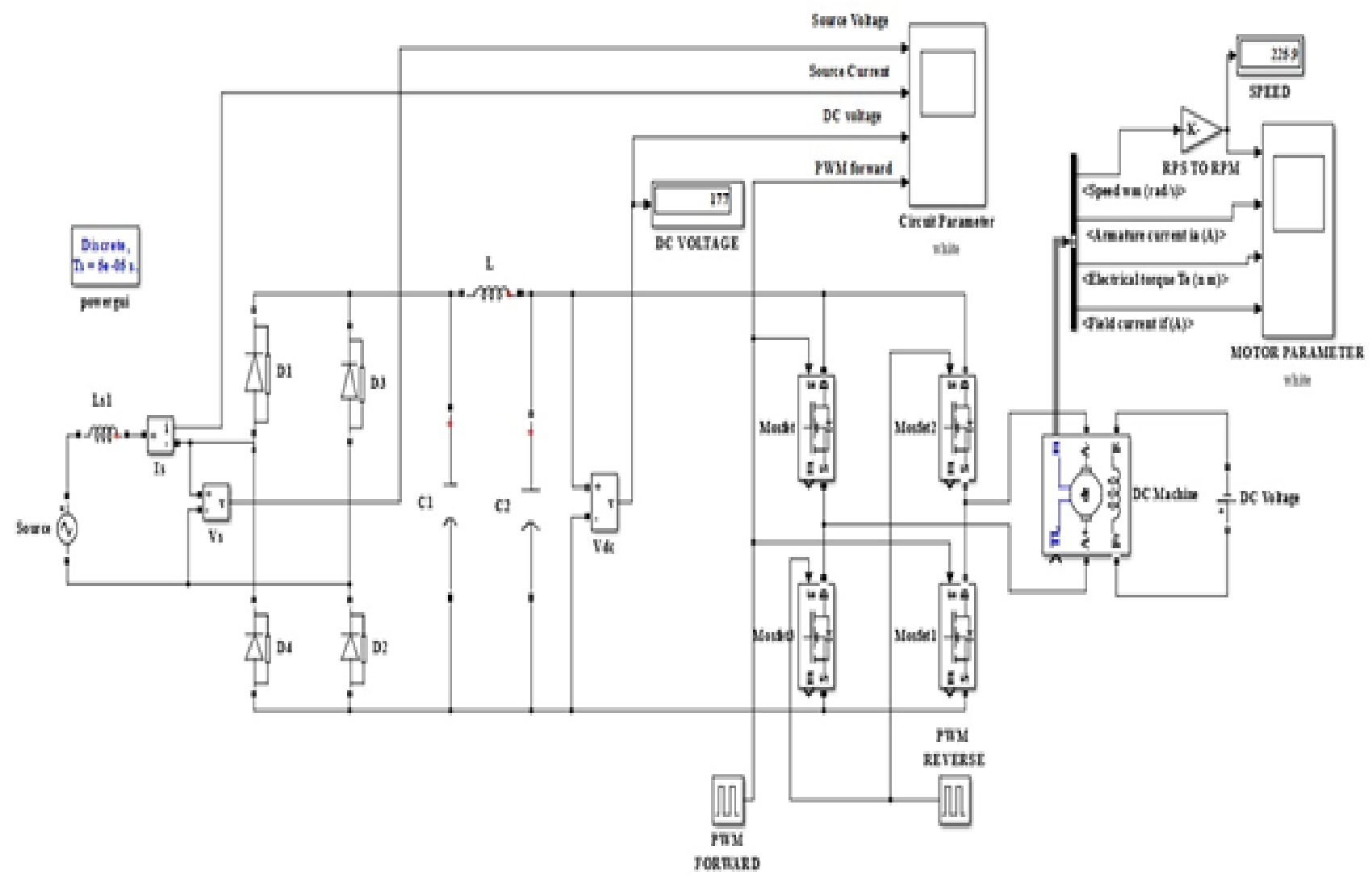

Fig 4 matlab simulation of mosfet h-bridge for dc drive

The simulation for the H-bridge Configuration along with diode rectifier is carried out and using MATLAB Simulink r2013a with discrete simulation type which has the solver ode23t (mod. Stiff/trapezoidal). Circuit for the same is shown in the FIGURE 4. DC machine is drawn by two of the MOSFETs at a time. 

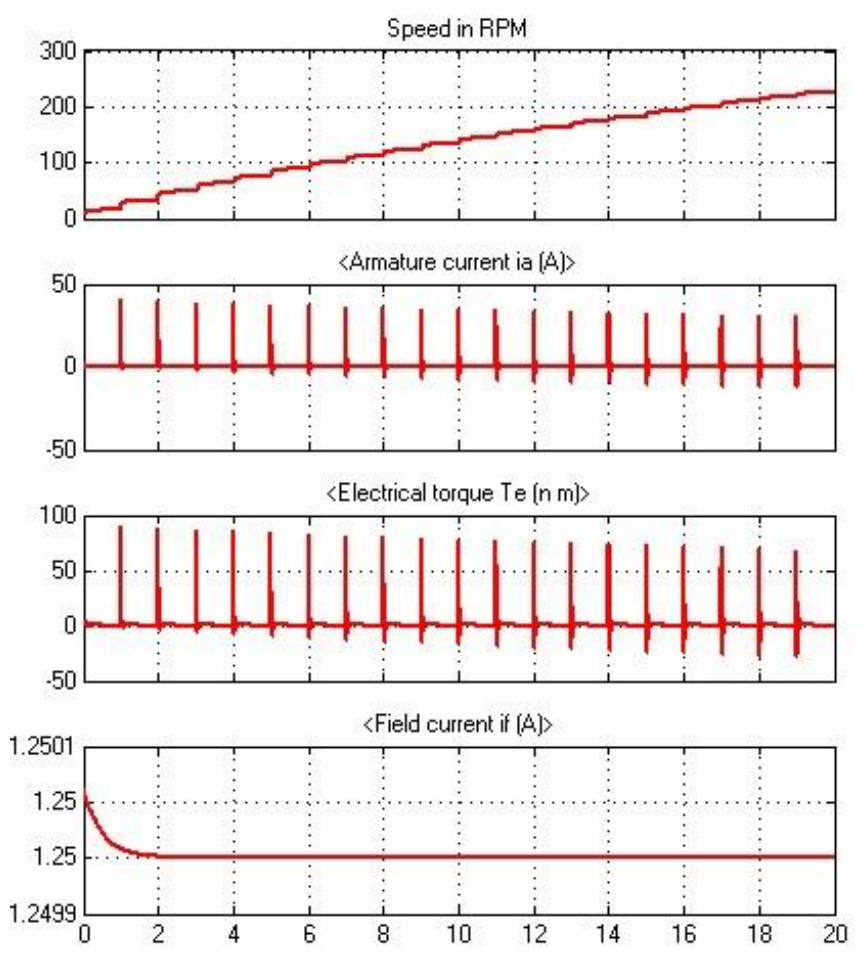

Fig 5 simulation results for forward operation of a motor

The simulation results for the motor parameters are shown in the FIGURE 5 with $50 \%$ duty cycle of the PWM. The waveform indicates the motor speed curve for the forward rotation of the motor, armature current curve, motor torque and field current curve. It is clearly indicated in the speed curve that the speed is increased in a step manner depending upon the PWM pulses. The PIC16f877a can be programed such that it generates PWM with various duty cycle accordingly the speed requirement.

\section{MICROCONTROLLER}

The PIC16f877a microcontroller has a following features: Only 35 single-word instructions to learn. Timer0:8-bit timer/counter with 8-bit prescaler. Timer1:16-bit timer/counter with prescaler can be incremented during Sleep via external Crystal/clock. Timer2: 8-bit timer/counter with 8bit period register, prescaler and postscaler. Two Capture, Compare, PWM (CCP) modules: Capture is of 16-bit with maximum resolution $12.5 \mathrm{~ns}$; Compare is of 16-bit with maximum resolution $200 \mathrm{~ns}$; PWM maximum resolution is 10bit, 100,000 erase/write cycle Enhanced Flash program memory typical, 1,000,000 erase/write cycle Data EEPROM memory typical Data. EEPROM Retention > 40 years. Selfreprogrammable under software control. [5]

\section{PWM TECHNIQUE}

A simplest method to control the rotation speed of DC motor is to control the driving voltage. The higher the voltage the higher the speed motor tries to reach. In many application simple voltage regulation would cause a lot of power loss in control circuit, so Pulse Width Modulation (PWM) is used in many DC motor controlling application. The rapid rising and falling edges ensure that the semiconductor power devices are turned on or turned off as fast as practically possible to minimize the switching transition time and the associated switching losses.

Although other considerations, such as parasitic ringing and Electromagnetic Interference (EMI) emission, may impose an upper limit on the turn-on and turn-off speed in practical situations, the resulting finite rise and fall time can be ignored in the analysis of PWM signals and processes in most cases.

The average value of voltage fed to the load is controlled by turning the switch between supply and load ON and OFF at a fast pace. The longer the switch is ON compared to the OFF periods, the higher the power supplied to the load is. The term duty cycle describes the proportion of ON time to the regular interval or period of time, a low duty cycle corresponds to low power, because the power is OFF for most of the time. Duty cycle is expressed in percent, $100 \%$ being fully ON.

PWM control technique also allows the switching frequency increment due to the simplicity of the control strategy and the availability of fast switching devices with reasonably power ratings [3].

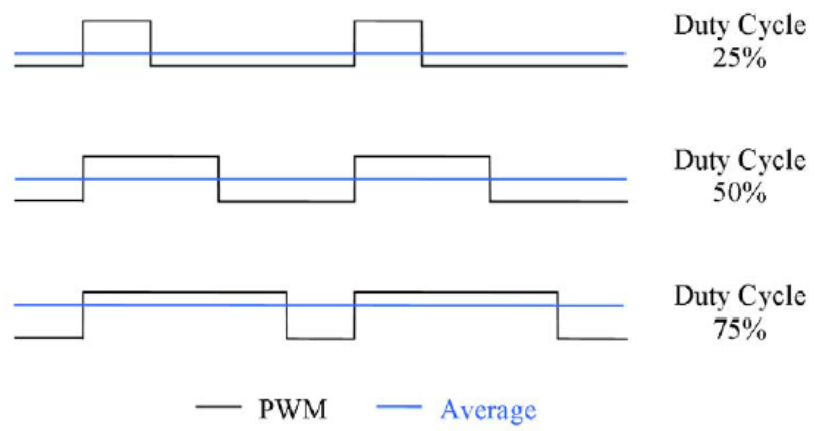

Fig 6 pwm waveform with different duty cycle

The PWM pulses are generated using PIC16F877A and the FIGURE 6 illustrates the waveforms for the various duty cycle. When this pulses are fed to the MOSFET gate terminal through a gate driver circuit, the average voltage between the two terminals of motor in $\mathrm{H}$-bridge are nearer to its maximum value.

The flowchart given in the FIGURE 7 illustrates a basic PWM generation using microcontroller. Here the PWM pulse will be 
given to feedback path and according to required duty cycle it will adjust required value of certain registers.

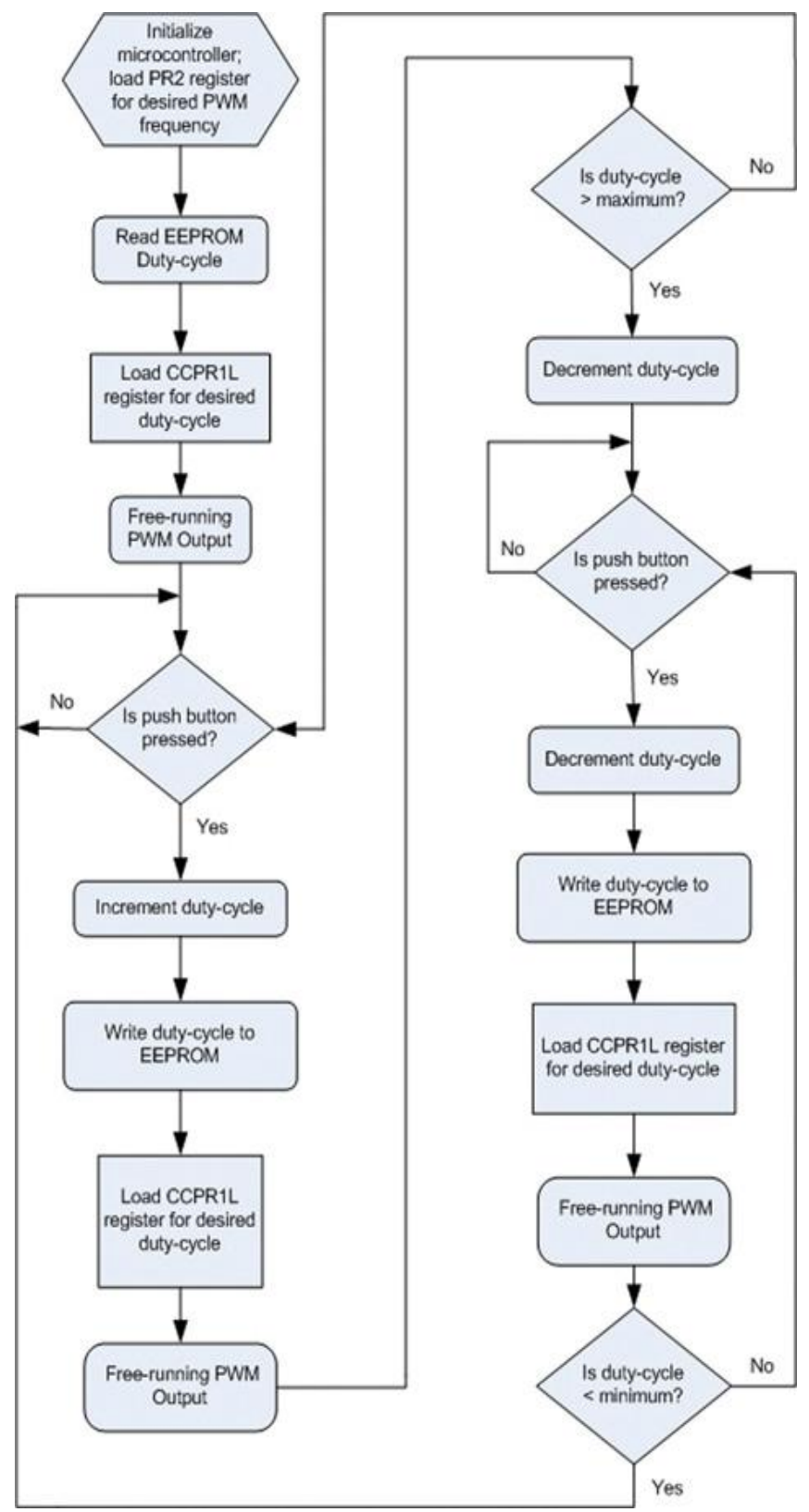

Fig 7 flowchart of pwm generation

\section{CONCLUSIONS}

In this paper, the drawbacks of a conventional Drive system are discussed following by the latest microcontroller based Drive system. PIC16F877A is preferable for the PWM based control system as it contains in-built PWM mode. Also the DC Drive system undergoes a good system response as the PWM control technique provides a soft starting to the motor so the starting high current can be limited which is supposed to be drawn by the motor from the mains. MOSFET H-bridge configuration provides bi-directional speed control by driving the appropriate pairs of MOSFETs.

\section{REFERENCES}

[1] Theraja, B. L., \& Theraja, A. k. (2005). A Textbook of Electrical Technology-volume 2. S.Chand.

[2] Dubey, G. K. (2001). Fundamentals of Electrical Drives. Narosa.

[3] Tamer H. Abdelhamid, "Performance of Single-Phase DC Drive System Controlled by Uniform PWM FullBridge DC-DC Converter" $10^{\text {th }}$ Mediterranean Electrotechnical Conference, MEleCon 2000, Vol. III

[4] Tantos, A. (2011). Retrieved from modularcircuits:http://www.modularcircuits.com/hbridge_secrets $1 . h$ tm

[5] (1998). Retrieved from Microchip: https://www.microchip.com/pagehandler/enus/products/picmicrocontrollers 